\title{
Novel 3-Dimensional K-Space segmented acquisition scheme (CENTRA-PLUS) for enhanced coronary imaging
}

\author{
Mita Patel ${ }^{1 *}$, Amit R Patel ${ }^{1}$, Hui Wang ${ }^{2}$, Donovan Gorre ${ }^{1}$, Roberto Lang ${ }^{1}$, Keigo Kawaji \\ From 19th Annual SCMR Scientific Sessions \\ Los Angeles, CA, USA. 27-30 January 2016
}

\section{Background}

Bright-blood coronary artery imaging by cardiovascular magnetic resonance remains a challenge due to several factors including coronary tortuosity, cardiac and respiratory motion, and high demands in spatial resolution and image contrast. Cartesian CENTRA-PLUS for high-resolution 3D k-space acquisition sorts the different regions of segmented k-space at different timepoints of the $\sim 5$ minute scan (figure 1). This strategy is well-suited for various acceleration methods including GRAPPA, compressed sensing, and navigator strategies for scan time reduction that conventional centric acquisition strategies do not easily allow. The aim of this study is to perform a direct comparison of CENTRAPLUS against the conventional centric acquisition for coronary CMR.

\section{Methods}

We studied ten healthy volunteers with both CENTRAPLUS and conventional centric acquisition strategies matched for all other acquisition parameters. Scans were performed on a $1.5 \mathrm{~T}$ Philips Achieva with a $5 \mathrm{ch}$ array $(\mathrm{TR}=4.4 \mathrm{~ms} ; \mathrm{TE}=1.9 \mathrm{~ms} ; \mathrm{FA}=90 ; 300 \times 300 \times 120$ $\mathrm{mm}^{3}$ acquired at $1.3-1.5 \mathrm{~mm}^{3}$ isotropic resolution, interpolated to $0.65 \times 0.65 \times 1.3 \mathrm{~mm}$, sensitive encoding parallel imaging $(\mathrm{r}=2))$. A fixed $5 \mathrm{~mm}$ gating window with no slice tracking was used. 5-point qualitative scores of coronary artery visualization $(1=$ not visible, $5=$ excellent) and quantitative parameters including Signal-toNoise-ratio (SNR) and Contrast-to-Noise-ratio (CNR) were evaluated using a fast noise scan for SENSE-accelerated analysis. Statistical analysis of SNR, CNR, and

${ }^{1}$ Cardiology, University of Chicago, Chicago, IL, USA

Full list of author information is available at the end of the article scan time was performed using Student's t-test and qualitative scores were assessed using Wilcoxon's SignedRank test.

\section{Results}

Qualitative scores for vessel visualization using CENTRA-PLUS were improved compared to the conventional centric acquisition strategy $(3.0 \pm 0.8$ vs $2.7 \pm 0.8$, $\mathrm{p}<0.05)$. In addition, there was an improvement in SNR of the right coronary artery (RCA) using CENTRAPLUS (98 \pm 16 vs $88 \pm 16, \mathrm{p}<0.002$ ) (figure 2 ). There was no significant difference in SNR of the left anterior descending (LAD) and left circumflex artery (LCX). The CNR of the RCA, LAD, and LCX were also comparable between both strategies $(\mathrm{p}=\mathrm{ns})$. Furthermore, there was no significant difference in scan time between the centric coronary MRA acquisition and CENTRA-PLUS (518 \pm $187 \mathrm{~s}$ vs $437 \pm 90 \mathrm{~s}, \mathrm{p}=\mathrm{ns}$ ) with matched navigator gating strategy.

\section{Conclusions}

CENTRA-PLUS is a promising acquisition scheme for coronary CMR resulting in improved image quality and SNR.

\section{Authors' details \\ ${ }^{1}$ Cardiology, University of Chicago, Chicago, IL, USA. ${ }^{2}$ Phillips Healthcare, Cleveland, $\mathrm{OH}$, USA.}

Published: 27 January 2016

doi:10.1186/1532-429X-18-S1-P112

Cite this article as: Patel et al:: Novel 3-Dimensional K-Space segmented acquisition scheme (CENTRA-PLUS) for enhanced coronary imaging. Journal of Cardiovascular Magnetic Resonance 2016 18(Suppl 1):P112. 

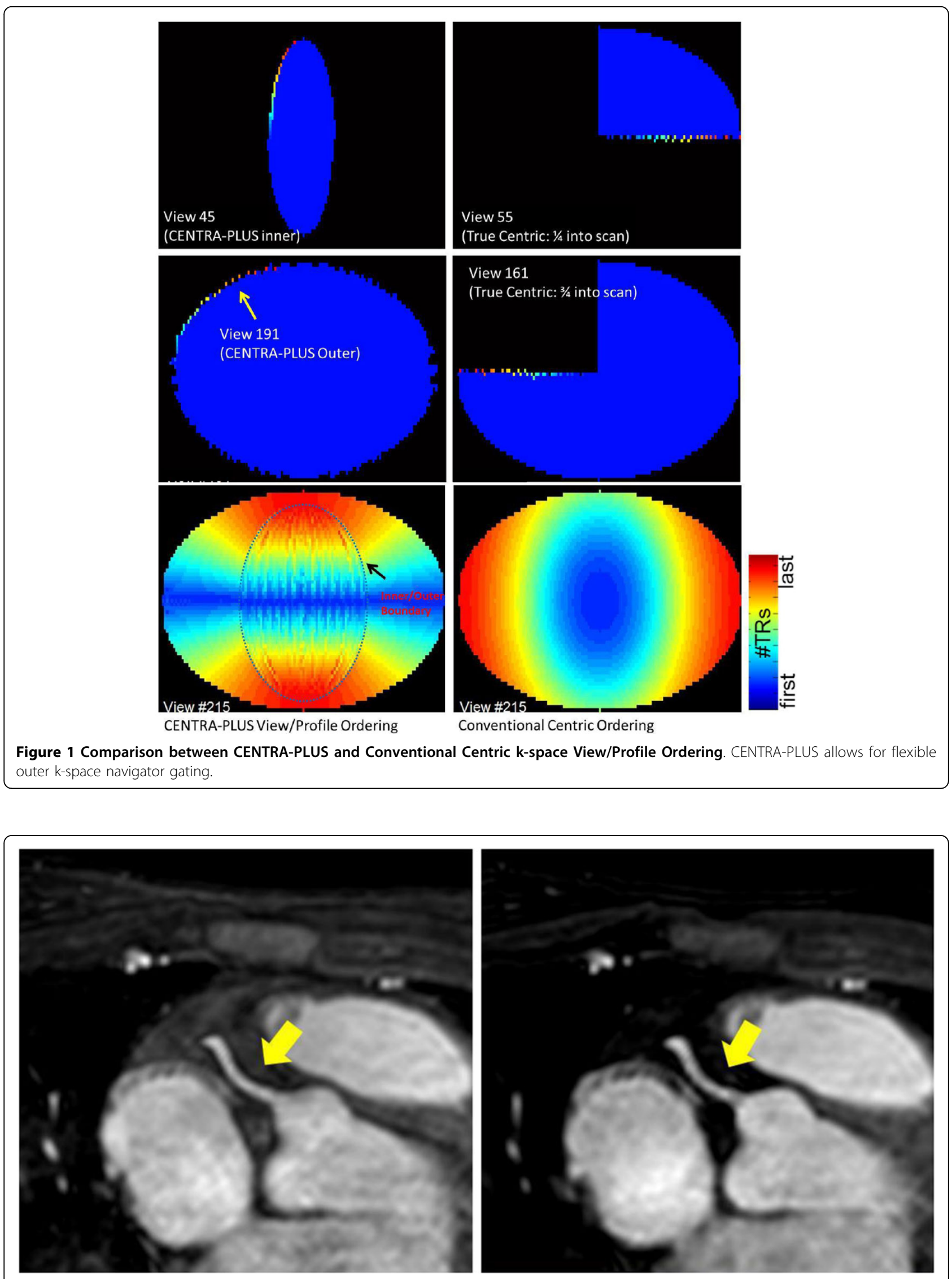

Figure 2 RCA visualization from one subject using: Left) CENTRA-PLUS and Right) Conventional Centric View/Profile Ordering. 